November 2, 2018

\title{
Lepton Universality, Rare Decays and Split Fermions
}

\author{
We-Fu Chang ${ }^{a}$, I-Lin $\mathrm{Ho}^{a, b}$ and John N. Ng${ }^{a}$ \\ ${ }^{a}$ TRIUMF Theory Group, 4004 Wesbrook Mall, Vancouver, B.C. V6T 2A3 CA \\ ${ }^{b}$ Physics Department, National Tsing-Hua University, \\ Hsinchu 30043, Taiwan, R.O.C.
}

\begin{abstract}
We investigate the constraint on the split fermions in extra dimensions by considering the universality of $W$ leptonic decays $W \rightarrow l_{i} \nu_{i}$, the charged lepton decays $l_{i} \rightarrow l_{j} \nu_{i} \bar{\nu}_{j}$, and the lepton flavor violating process $l_{i} \rightarrow \bar{l}_{j} l_{k} l_{h}$ where $l_{i}=e, \mu$ or $\tau$. For the Standard Model (SM) background of $W \rightarrow l_{i} \nu_{i}$, we extended the one loop quantum correction to include effects of order $m_{l}^{2} / M_{W}^{2}$ and the Higgs mass dependence. We find that in general the split fermion scenarios give rise to a 4D effective Yukawa matrix of the Kaluza-Klein Higgs bosons is misaligned with respect to the fermion mass matrix. This holds true also for gauge bosons as well. This leads to decays of $l_{i} \rightarrow \bar{l}_{j} l_{k} l_{h}$ at tree level and muonium antimuonium conversion. Interestingly the leptonic universality of $W$ boson decays are not affected at this level.
\end{abstract}

PACS numbers: $11.25 . \mathrm{Mj}, 12.60 .-\mathrm{i}, 11.10 . \mathrm{Kk}$ 


\section{Introduction}

Recently new avenues of exploring physics beyond the Standard Model(SM) have opened up by assuming that there exists large extra dimensions beyond the four we are familiar with [1], [2], [3]. The earlier investigations have the graviton and possibly SM gauge singlet particles such as the right-handed neutrinos are allowed to propagate in the extra dimensions whereas the particles that have SM charges are confined to a 4 dimensional hypersurface known as the $\mathrm{TeV}$ or the $\mathrm{SM}$ brane. This picture can also provide a natural geometrical understanding of the hierarchy of fermion masses by postulating that the chiral fermions of the SM are localized at different points in the extra dimensions [4]; i.e. they are split from each other. By the same token different families of fermions also occupy different points in bulk space. The localization of a chiral fermion is represented by a Gaussian wave function in the extra dimension $y$. The mass of a fermion is generated via a five dimensional Yukawa term. In four dimensions, after integrating out $y$, a small Yukawa coupling arises due to the small overlap of the wave functions of the leftand right- handed components of a fermion. In this way a hierarchy in the effective 4D Yukawa couplings is obtained without invoking new symmetries. A detail model for the observed quark and lepton masses in terms of their displacements in $y$ has been given in [5]. Besides offering a new vista on the Yukawa coupling hierarchy this scenario also points to a novel way of looking at the question of gauge coupling universality. Historically, the branching ratio $(\mathrm{Br})$ of $\pi \rightarrow e \nu / \pi \rightarrow \mu \nu$ provided the crucial evidence that the charged weak current couples with the same strength to the first two lepton families. This universality study has since been extended to leptonic $\tau$ decays and also to the leptonic branching ratios of the $W$ boson. These are cornerstones that support the SM and they are very accurately predicted in the 
SM. An example is the $\operatorname{Br}\left(W \rightarrow l_{i} \nu / W \rightarrow l_{j} \nu\right)=1+O(\alpha)$ where $l_{i}=e, \mu$, or $\tau$. In the SM the deviation from unity is a function of lepton masses and the lepton energy cut used in a given experiment [13]. The dependence on the unknown Higgs boson mass is very weak. As a by-product of our investigation we will give the complete 1-loop SM result. We feel that it is very important to examine how proposed new physics will altered these predictions.

To see more quantitatively how these various purely leptonic reactions can be used to probe the split fermion scenario we study the simplest model with the minimal SM in 5D. The chiral fermions are confined at different positions in the extra dimension. The exact mechanism of localization is not important to our study and we shall leave it open. The $S U(2) \times U(1)$ gauge bosons and the Higgs doublet are allowed to propagate in the full 5D bulk. The model is compactified in a $S_{1} / Z_{2}$ orbifold of radius $R$ with the appropriate boundary conditions so as to preserve the successes of the 4D SM. An interesting generic feature of the split fermions scenario is the existence of effective flavor changing neutral currents which we shall demonstrate are related to the separation between two chiral fermions belonging to different families. This is first noticed in [6] for the KK gauge bosons in the model. We extend this to both the neutral and the charged KK Higgs bosons. In this paper we concentrate on the issue of lepton flavor violation (LFV) interactions partly because they involve less theoretical uncertainties. We will also concentrate on tree level predictions of charged lepton decays. We will not discuss the many issues related to neutrino mass in the bulk world scenario even though they are very interesting. Neutrino masses can be made natural within the framework of extra dimensions [8] by introducing one [9] or more $\nu_{R}$ and phenomenological studies of the properties of bulk neutrinos can be found in [10] and references therein. Localizing the right- 
handed bulk neutrinos at different points in the extra dimension is performed in [11] and [14]. The latter reference also discussed lepton flavor violation in a general way which is different from our treatment. After acknowledging this we shall assume that neutrino masses are due to yet unknown $4 \mathrm{D}$ new physics and its phenomenology is beyond the scope of this paper.

In the quark sector an extensive numerical study of the quark mass matrices and their mixings is given in [5] whereas the issue of $\mathrm{CP}$ violation has been investigated in [7]. Furthermore supersymmetry has also been incorporated in this scenario in [11]. A related but different scheme using multilocation is given in [12]

This paper is organized as followed. In Sec 2 we give the details of the 5D SM model and obtain the 4D effective interactions after integrating out the extra dimension. The Feynman rules for the 4D interactions are summarized in the Appendix. Sec.3 gives the phenomenology of both normal and rare decays of charged leptons. In particular the decay of $\mu$ to 3 e provides the strongest constrain to the parameters of the model. In Sec 4 we compare the tests of universality using leptons and on shell W-boson decay. They are shown to be complementary and will be an important task for the Large Hadron Collider currently under construction. Finally we give our conclusions in Sec 5 .

\section{5D SM Model with Split Leptons}

The model we employed is the 5D SM similar to that introduced in Ref. [6] augmented by the distributions of chiral fermions located at different points in the extra dimension, $y$. It is a crucial assumption that the left-handed $(L)$ lepton doublet is separated from the right-handed $(R)$ lepton. For the minimal matter content 
of the SM ignoring neutrino mass there are six independent locations $y_{i}^{a}$ where we use $i, j, k$ for family indices and $a, b \in\{L, R\}$ stand for chiralities. One of these can be chosen as the origin. The $4 \mathrm{D}$ effective theory is obtained by compactifying the bulk fields on a $S_{1} / Z_{2}$ orbifold where $S_{1}$ is a circle define by $-\pi R \leq y \leq \pi R$. Strictly speaking $R$ is a free parameter and is bounded by experiments. We shall assume that $R \lesssim(300 \mathrm{GeV})^{-1}$ for the sake of phenomenological interest. Then we implement the idea that chiral fermions can be trapped at topological domain wall in such a setting [15] and also at different locations [4], [16]. The zero mode of a fermion is chiral and is given a narrow Gaussian distribution in $y$. We adopt a universal Gaussian width $\sigma$ for all the fermions. We use the notation that the coordinates in Minkowski space is denoted by $x^{\mu}, \mu=\{0 \cdots 3\}$ and in bulk space by $x^{M}, M=\{0 \cdots 3, y\}$. Also the fifth Dirac matrix is chosen to be $\gamma^{y}=i \gamma_{5}$.

The 5D SM Lagrangian is given by

$$
\begin{aligned}
\mathcal{L}_{5}= & -\frac{1}{4} F^{M N} F_{M N}-\frac{1}{4} G^{(c), M N} G_{M N}^{(c)}+\overline{L^{\prime}}(x, y) i \gamma^{M} D_{M} L^{\prime}(x, y) \\
& +\left(D_{M} \Phi(x, y)\right)^{\dagger}\left(D^{M} \Phi(x, y)\right)-\kappa R\left(|\Phi(x, y)|^{2}-\frac{v_{b}^{3}}{2}\right)^{2} \\
& -\sqrt{2 \pi R} f_{i j}{\overline{L^{\prime}}}_{i}(x, y) \Phi(x, y) E_{j}^{\prime}(x, y)+\text { h.c. }+\cdots
\end{aligned}
$$

where $L^{\prime}$ and $E^{\prime}$ are respectively the $S U(2)$ doublet and singlet lepton fields. $\Phi$ is the bulk Higgs field and $v_{b}$ is its vacuum expectation value (VEV). For simplicity, we take a universal Yukawa coupling $f$ which is of order one for $f_{i j}$. Also, as in the SM,

$$
\begin{aligned}
D_{M} & =\partial_{M}-i g_{2} \sqrt{2 \pi R} \frac{\tau^{c}}{2} B_{M}^{c}-i g_{1} \sqrt{2 \pi R} \frac{Y}{2} A_{M}, \\
F_{M N} & =\partial_{M} A_{N}-\partial_{N} A_{M} \\
G_{M N}^{(c)} & =\partial_{M} B_{N}^{c}-\partial_{N} B_{M}^{c}+\sqrt{2 \pi R} g_{2} \epsilon^{c d e} B_{M}^{d} B_{N}^{e}
\end{aligned}
$$


and $A, B$ stand for the $U(1)$ hyper charge and $S U(2)$ gauge fields. In this convention, $Q=T_{3}+Y / 2$. Note that the mass dimensions of various quantities are: $[\Psi]=2,[\Phi]=\frac{3}{2},\left[g_{1}\right]=\left[g_{2}\right]=0,[\kappa]=0$ and $[f]=0$.

In our study we can ignore KK excitations of the fermions but will keep the KK excitations of Higgs and gauge bosons. For $\sigma \ll R$, the chiral zero mode of a fermion field $\Psi_{i}^{a}$ located at $y_{i}^{a}$ can be normalized to

$$
\Psi_{i}^{a}(x, y) \sim \frac{1}{\pi^{\frac{1}{4}} \sigma^{\frac{1}{2}}} \Psi_{i}^{a}(x) e^{-\frac{\left(y-y_{y^{2}}\right)^{2}}{2 \sigma^{2}}} .
$$

The product of two fermion fields can be approximately replaced by

$$
\bar{\Psi}_{i}^{a}(x, y) \Psi_{j}^{b}(x, y) \sim \exp \left(-\frac{\left(\triangle_{i j}^{a b}\right)^{2}}{4 \sigma^{2}}\right) \delta\left(y-\bar{y}_{i j}^{a b}\right) \bar{\Psi}_{i}^{a}(x) \Psi_{j}^{b}(x)
$$

where $\bar{y}_{i j}^{a b}=\left(y_{i}^{a}+y_{j}^{b}\right) / 2$ is their average positions and $\triangle_{i j}^{a b}=y_{i}^{a}-y_{j}^{b}$. It is known [17] that the 5D SM where all fields propagate in the full bulk there is conservation of $\mathrm{KK}$ number in the $4 \mathrm{D}$ effective theory due to momentum conservation. It is interesting that when a Gaussian profile is given to the fermion filed the usual KK number conservation now is replaced by a suppression factor

$$
\cos \frac{n y_{i}}{R} e^{-\frac{n^{2} \sigma^{2}}{4 R^{2}}} \text {. }
$$

This is because

$$
\int_{-\pi R}^{\pi R} d y \cos \frac{n y}{R} \frac{1}{\pi^{1 / 2} \sigma} e^{-\frac{\left(y-y_{i}\right)^{2}}{\sigma^{2}}} \cong \cos \frac{n y_{i}}{R} e^{-\frac{n^{2} \sigma^{2}}{4 R^{2}}} .
$$

This is understood because that the Gaussian localized wave function serves as the fifth momentum 'reservoir' that compensates the momentum carried by bulk gauge boson and thus maintains the conservation of momentum. Now it is possible to have vertices with only one non-zero KK mode. Since we expect $\sigma \ll R$ the exponential factor is almost unity. 
The fermion and gauge boson masses are generated by the VEV of bulk Higgs which we write as

$$
\Phi(x, y)=\left(\begin{array}{c}
h^{+}(x, y) \\
\frac{1}{\sqrt{2}}\left(v_{b}^{\frac{3}{2}}+\phi^{0}(x, y)\right)
\end{array}\right) .
$$

The scalar field is taken to be even under $Z_{2}$ and the KK decomposition is given by

$$
\phi^{0}(x, y)=\frac{1}{\sqrt{2 \pi R}}\left(\phi_{0}^{0}(x)+\sqrt{2} \sum_{n=1}^{\infty} \phi_{n}^{0}(x) \cos \frac{n y}{R}\right) .
$$

The zero mode $\phi_{0}^{0}(x)=h_{0}^{0}+i \chi_{0}$ is identified as the SM Higgs boson, $h_{0}^{0}$, and its Goldstone partner $\chi_{0}^{0}$. The KK tower also contains a real and imaginary part given by $\phi_{n}^{0}=h_{n}^{0}+i \chi_{n}^{0}$. A similar expression holds for the gauge fields but their fifth component are assigned to be odd under $Z_{2}$, so as to prevent the presence of the unwanted zero modes at the orbifold fix point. This leads explicitly to the following expansion for gauge fields

$$
\begin{array}{r}
A^{\mu}(x, y)=\frac{1}{\sqrt{2 \pi R}}\left(A_{0}^{\mu}(x)+\sqrt{2} \sum_{n=1}^{\infty} A_{n}^{\mu}(x) \cos \frac{n y}{R}\right), \\
A^{4}(x, y)=\frac{1}{\sqrt{\pi R}}\left(\sum_{n=1}^{\infty} A_{n}^{4}(x) \sin \frac{n y}{R}\right) .
\end{array}
$$

As in $4 \mathrm{D}$ gauge theory one has to fix a gauge in a given calculation. The 5D generalization of covariant gauge fixing Lagrangians are

$$
\begin{aligned}
\mathcal{L}_{G F}= & -\frac{1}{2 \alpha}\left(\partial_{M} P^{M}\right)^{2}-\frac{1}{\xi}\left|\partial_{M} W^{+, M}-i \xi \frac{g_{5} \cos \theta_{W} v_{b}^{3 / 2}}{2} h^{+}\right|^{2} \\
& -\frac{1}{2 \eta}\left(\partial_{M} Z^{M}-\eta \frac{g_{5} v_{b}^{3 / 2}}{2} \chi^{0}\right)^{2}
\end{aligned}
$$

where $\chi^{0}=\operatorname{Im}\left(\phi^{0}\right)$ is the pseudoscalar would be Goldstone boson, and $\alpha, \xi, \eta$ are the gauge parameters for the photon $(P), \mathrm{W}$ and $\mathrm{Z}$ bosons respectively. Also $g_{5}=$ 
$\sqrt{2 \pi R} \sqrt{g_{1}^{2}+g_{2}^{2}} \equiv \sqrt{2 \pi R} g$. Combining $\mathcal{L}_{5}$ and $\mathcal{L}_{G F}$ and integrating over $y$, we get the 4D effective Lagrangian. One finds that the usual tree level SM relations still held,

$$
\begin{array}{r}
g=\sqrt{g_{1}^{2}+g_{2}^{2}}, \quad e=g_{2} \sin \theta_{W}, \quad \tan \theta_{W}=\frac{g_{1}}{g_{2}} \\
M_{Z}=\frac{g \sqrt{2 \pi R} v_{b}^{3 / 2}}{2}, \quad M_{W}=M_{Z} \cos \theta_{W} .
\end{array}
$$

It is straightforward to obtain the $n^{\mathrm{th}}-\mathrm{KK}$ gauge boson masses. They are: $M_{\gamma, n}^{2}=$ $n^{2} / R^{2} ; M_{W, n}^{2}=M_{W}^{2}+n^{2} / R^{2} ; M_{Z, n}^{2}=M_{Z}^{2}+n^{2} / R^{2}$. For the Higgs bosons one has $M_{H}^{2}=\kappa R v_{b}^{3}$ and $M_{h^{0}, n}^{2}=M_{H}^{2}+n^{2} / R^{2}$. In this model, the fifth component of gauge fields behave like spin-0 particles and their masses are $M_{A^{4}, n}^{2}=n^{2} /\left(\alpha R^{2}\right)$, $M_{Z^{4}, n}^{2}=M_{Z}^{2}+n^{2} /\left(\eta R^{2}\right)$ and $M_{W^{4}, n}^{2}=M_{W}^{2}+n^{2} /\left(\xi R^{2}\right)$. They couple to the SM gauge bosons on the brane but not couple to the brane fermions through gauge interaction. Another way to see the absence of couplings between the fifth components and brane fermions is that the interaction $\bar{\Psi} A^{4} \Psi$ is odd under the orbifolding $Z_{2}$ parity.

The Higgs sector is same as in the SM,

$$
-\sqrt{2 \pi R} f\left[\bar{\nu}_{L i} h^{+}+\frac{1}{\sqrt{2}}\left(v_{b}^{3 / 2}+h^{0}+i \chi^{0}\right) \bar{e}_{L i}\right] e_{R j}+H . c .
$$

The masses of the would-be-Goldstone bosons and their KK partners are: $M_{\chi^{0}, n}^{2}=$ $\eta M_{Z}^{2}+n^{2} / R^{2}$ and $M_{h^{+}, n}^{2}=\xi M_{W}^{2}+n^{2} / R^{2}$. In the $4 \mathrm{D}$ effective Lagrangian the following mixing terms also appear

$$
\begin{aligned}
& \frac{n}{R} \frac{1-\alpha}{\alpha}\left(\partial^{\mu} P_{n}^{4}\right) P_{n \mu}+\frac{n}{R} \frac{1-\eta}{\eta}\left(\partial^{\mu} Z_{n}^{4}\right) Z_{n \mu} \\
& +\frac{n}{R} \frac{1-\xi}{\xi}\left(\partial^{\mu} W_{n}^{4+} W_{n \mu}^{-}+\partial^{\mu} W_{n}^{4-} W_{n \mu}^{+}\right) .
\end{aligned}
$$

Clearly by choosing the Feynman gauge, $\alpha=\xi=\eta=1$, one can eliminate the mixing terms. This is the most convenient gauge for calculating physical processes. We summarize the Feynman rules we employ in the Appendix. 
The effective 4D Yukawa coupling for charged leptons is

$$
\mathcal{L}_{Y}=-f e^{-\frac{\left(\triangle_{i j}^{L R}\right)^{2}}{4 \sigma^{2}}} \overline{L_{i}^{\prime}}(x)\left[\sqrt{\pi R} v_{b}^{\frac{3}{2}}+\frac{\phi_{0}^{0}}{\sqrt{2}}+\sum_{n=1} \phi_{n}^{0} \cos \frac{n \overline{y_{i j}^{L R}}}{R} e^{-\frac{n^{2} \sigma^{2}}{4 R^{2}}}\right] E_{j}^{\prime}(x)+H . c .
$$

The mass matrix is readily seen to be

$$
\mathcal{M}_{i j}=f \sqrt{\pi R} v_{b}^{\frac{3}{2}} e^{-\frac{\left(\triangle_{i j}^{L} R\right)^{2}}{4 \sigma^{2}}}=f \frac{\sqrt{2} M_{W}}{g_{2}} e^{-\frac{\left(\triangle_{i j}^{L R}\right)^{2}}{4 \sigma^{2}}} \simeq f m_{t} e^{-\frac{\left(\triangle_{i j}^{L R}\right)^{2}}{4 \sigma^{2}}} .
$$

Temporarily suppressing family indices, the mass matrix is diagonalized by a biunitary transformation as follows

$$
M_{\text {diag }}=V^{L \dagger} M V^{R}, \quad L^{\prime}(x)=V^{L} L(x), \quad E^{\prime}(x)=V^{R} E(x)
$$

where $L(x)$ and $E(x)$ are mass eigenstates.

It is easy to see that this diagonalization also rotates away the off-diagonal coupling of fermions to the Higgs zero mode; i.e. the SM Higgs to fermion couplings remain flavor diagonal. Furthermore, the SM gauge bosons fermion couplings are also flavor diagonal. However, the Higgs KK modes will couple different mass eigenstate fermions as displayed in

$$
\mathcal{L}=-\bar{L}_{i}\left(m_{i}+\frac{g_{2} m_{i}}{2 M_{W}} \phi_{0}^{0}\right) E_{i}-\sum_{n=1} \lambda_{i j, n}^{L R} \bar{L}_{i} E_{j} \phi_{n}^{0}+\text { h.c. }
$$

where

$$
\lambda_{i j, n}^{L R}=f V_{k i}^{L *} \exp \left(-\frac{\left(\triangle_{k l}^{L R}\right)^{2}}{4 \sigma^{2}}\right) \cos \frac{n \bar{y}_{k l}^{L R}}{R} V_{l j}^{R} e^{-\frac{n^{2} \sigma^{2}}{4 R^{2}}} .
$$

In other words one cannot simultaneously diagonalize the fermion mass matrix and the Yukawa matrix of the KK Higgs-fermion couplings due to the presence of the cosine terms. Similar flavor nondiagonal couplings are induced for the KK excitations of the $W$ and $Z$ bosons as well. After some algebra, the effective $4 \mathrm{D}$ charged current Lagrangian can be cast in the form

$$
\mathcal{L}_{\text {eff }}^{C C}=g_{2} \overline{L_{i}}\left[\gamma^{\mu} \frac{\tau^{+}}{\sqrt{2}}\left(\delta_{i j} W_{0, \mu}^{+}+\sum_{n=1} W_{n, \mu}^{+}(x) U_{i j}^{L(n)}\right)\right] L_{j}+\text { h.c. }
$$


and the neutral Lagrangian is

$$
\begin{aligned}
\mathcal{L}_{\text {eff }}^{N C} & =\frac{g_{2} g_{L}}{\cos \theta_{W}} \overline{L_{i}}\left[\gamma^{\mu}\left(\delta_{i j} Z_{0, \mu}+\sum_{n=1} Z_{n, \mu}(x) U_{i j}^{L(n)}\right)\right] L_{j} \\
& +\frac{g_{2} g_{R}}{\cos \theta_{W}} \overline{E_{i}}\left[\gamma^{\mu}\left(\delta_{i j} Z_{0, \mu}+\sum_{n=1} Z_{n, \mu}(x) U_{i j}^{R(n)}\right)\right] E_{j} \\
& + \text { h.c }
\end{aligned}
$$

where $g_{L / R}=T_{3, L / R}-Q_{L / R} \sin ^{2} \theta_{W}$. And

$$
\begin{aligned}
U_{i j}^{L(n)} & =\sqrt{2} \sum_{k=1}^{3} V_{k i}^{L *} \cos \frac{n y_{k}^{L}}{R} V_{k j}^{L} e^{-\frac{n^{2} \sigma^{2}}{4 R^{2}}}, \\
U_{i j}^{R(n)} & =\sqrt{2} \sum_{k=1}^{3} V_{k i}^{R *} \cos \frac{n y_{k}^{R}}{R} V_{k j}^{R} e^{-\frac{n^{2} \sigma^{2}}{4 R^{2}}} .
\end{aligned}
$$

The very same mixings $U_{i j}^{L(n)}$ and $U_{i j}^{R(n)}$ are also associate with the KK photon.

Clearly the KK excitations of the photon, the $Z$ boson, and the Higgs boson will all induce tree level lepton flavor violation processes. We will discuss their contributions in detail in the next section.

\section{Constraint on the fermion locations}

Equipped with the Feynman rules given we can proceed to discuss the phenomenology of charged lepton decays. Consider first classic case of muon decay into an electron and a pair of neutrinos. At the tree level the SM has only one amplitude involving $W$ boson will now have contributions also from its KK excitations. The split fermion scenario also adds the contributions from KK excitations of the $Z$ (see Eq.18) and the charged Higgs boson. Also a sum over the neutrinos in the final state is taken. Similar modifications to the usual discussions of rare decays also occurs and they are systematically presented in the following subsections. 


\subsection{Lepton Universality}

In terms of mass eigenstate the effective $4 \mathrm{D}$ charge current interaction is given by Eq.(17). Since we only have one bulk Higgs field there is no mixing between physical $W$ boson, which is the zero mode, and its KK excitation. Therefore, it is universally coupled to the lepton families. We conclude that lepton universality tested by ratios of the leptonic width of the $W$ boson will remain at the SM values at the lowest level.
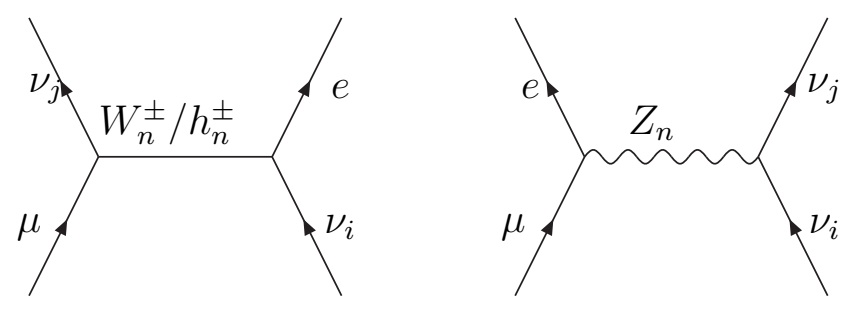

Figure 1: Contribution of KK excitations to $\mu$ decay

On the other hand for the classic decay of $\mu \rightarrow e \nu \bar{\nu}$, where all the possible virtual KK modes also participate, information on the $y$-dependence can be gleamed. Neglecting the electron mass we get

$$
\begin{aligned}
-\mathcal{M} & =\left(\frac{g_{2}}{\sqrt{2}}\right)^{2}\left[\frac{\delta_{i 1} \delta_{j 2}}{M_{W}^{2}}+\sum_{i, j, n} \frac{U_{i 1}^{L(n)} U_{j 2}^{L(n) *}}{M_{W, n}^{2}}\right]\left(\bar{\nu}_{j} \gamma_{L}^{\alpha} \mu\right)\left(\bar{e} \gamma_{L, \alpha} \nu_{i}\right) \\
& -\sum_{i, j, n} \frac{2 \lambda_{i 2, n}^{L R} \lambda_{j 1, n}^{L R *}}{M_{h^{+}, n}^{2}}\left(\bar{\nu}_{j} \mu\right)\left(\bar{e} \nu_{i}\right) \\
& +g_{L}^{\nu} g_{L}^{e}\left(\frac{g_{2}}{\cos \theta}\right)^{2} \sum_{i, j, n} \frac{U_{12}^{L(n)} U_{j i}^{L(n) *}}{M_{Z, n}^{2}}\left(\bar{\nu}_{j} \gamma_{L}^{\alpha} \nu_{i}\right)\left(\bar{e} \gamma_{L, \alpha} \mu\right) \\
& +g_{L}^{\nu} g_{R}^{e}\left(\frac{g_{2}}{\cos \theta}\right)^{2} \sum_{i, j, n} \frac{U_{12}^{R(n)} U_{j i}^{L(n) *}}{M_{Z, n}^{2}}\left(\bar{\nu}_{j} \gamma_{L}^{\alpha} \nu_{i}\right)\left(\bar{e} \gamma_{R, \alpha} \mu\right)
\end{aligned}
$$




$$
\begin{aligned}
\equiv & \left(\frac{g_{2}^{2}}{2 M_{W}^{2}}\right)\left\{\left(1+a_{1} R^{2} M_{W}^{2}\right)\left(\bar{\nu} \gamma_{L}^{\alpha} \mu\right)\left(\bar{e} \gamma_{L, \alpha} \nu\right)\right. \\
& -a_{2} M_{W}^{2} R^{2}\left(\bar{\nu} \mu_{R}\right)\left(\bar{e}_{R} \nu\right) \\
& \left.+a_{3} M_{W}^{2} R^{2}\left(\bar{\nu} \gamma_{L}^{\alpha} \mu\right)\left(\bar{e} \gamma_{L, \alpha} \nu\right)-2 a_{4} M_{W}^{2} R^{2}\left(\bar{\nu} \mu_{R}\right)\left(\bar{e}_{R} \nu\right)\right\}
\end{aligned}
$$

The Fierz transformation have been used to get the last expression. The coefficients $a_{i, j, k}$ are the result of summing over all neutrino species. Explicitly,

$$
\begin{array}{r}
a_{1} \sim \sum_{i, j, n=1} \frac{U_{j 2}^{L(n)} U_{1 i}^{L(n) *}}{n^{2}}, a_{2} \sim \sum_{i, j, n=1} \frac{4 \lambda_{j 2, n}^{L R} \lambda_{i 1, n}^{L R *}}{g_{2}^{2} n^{2}}, \\
a_{3} \sim\left(\frac{2 g_{L}^{\nu} g_{L}^{e}}{\cos ^{2} \theta}\right) \sum_{i, j, n=1} \frac{U_{12}^{L(n)} U_{i j}^{L(n) *}}{n^{2}}, a_{4} \sim\left(\frac{2 g_{L}^{\nu} g_{R}^{e}}{\cos ^{2} \theta}\right) \sum_{i, j, n=1} \frac{U_{12}^{R(n)} U_{i j}^{L(n) *}}{n^{2}} .
\end{array}
$$

We define the process dependent Fermi constant $G_{\mu}$ as

$$
\frac{4 G_{\mu}}{\sqrt{2}}=\frac{g_{2}^{2}}{2 M_{W}^{2}}\left[\left(1+R^{2} M_{W}^{2}\left(a_{1}+a_{3}\right)\right)^{2}+\left(\frac{a_{2}+2 a_{4}}{2}\right)^{2} R^{4} M_{W}^{4}\right]^{\frac{1}{2}}
$$

The square bracket gives the modification to the SM Fermi coupling constant, $G_{S M, F}=\sqrt{2} g_{2}^{2} / 8 M_{W}^{2}$ and also generalizes the usual KK result [18. Eq. 20) reveals that the Michel parameters $\rho$ and $\delta$ will have the SM value of $3 / 4$. This is easily seen in the charge retention mode. On the other hand we have

$$
\eta \simeq-\frac{\left(a_{2}+2 a_{4}\right) R^{2} M_{W}^{2}}{2}
$$

Thus, we expect a deviation from the SM value of $\eta=0$. This in turn leads to the following prediction for the partial decay width of a charged lepton into a purely leptonic channel $l_{i} \rightarrow l_{j}+\bar{\nu} \nu$ in this model :

$$
\begin{aligned}
\Gamma\left(l_{i}\right. & \left.\rightarrow l_{j}+\bar{\nu} \nu\right)=\frac{m_{i}^{5} G_{i j}^{2}}{192 \pi^{3}}\left\{1-8 \alpha_{i j}^{2}+8 \alpha_{i j}^{6}-\alpha_{i j}^{8}-24 \alpha_{i j}^{4} \ln \alpha_{i j}\right. \\
& \left.+4 \eta_{i j} \alpha_{i j}\left(1+9 \alpha_{i j}^{2}-9 \alpha_{i j}^{4}-\alpha_{i j}^{6}\right)+48 \eta_{i j} \alpha_{i j}^{3}\left(1+\alpha_{i j}^{2}\right) \ln \alpha_{i j}\right\}
\end{aligned}
$$


where $\alpha_{i j}=\frac{m_{j}}{m_{i}}, G_{i j}$ represents the specific Fermi constant for the process $l_{i} \rightarrow$ $l_{j}+\bar{\nu} \nu$. Expand in powers of $M_{W}^{2} R^{2}$ and keeping the lowest order we obtain

$$
\begin{aligned}
\Gamma\left(l_{i} \rightarrow l_{j}+\bar{\nu} \nu\right) \sim & \frac{m_{i}^{5} G_{S M, F}^{2}}{192 \pi^{3}}\left[1-8 \alpha_{i j}^{2}+8 \alpha_{i j}^{6}-\alpha_{i j}^{8}-24 \alpha_{i j}^{4} \ln \alpha_{i j}\right. \\
& \left.+2\left(a_{1}+a_{3}\right) M_{W}^{2} R^{2}-2 \alpha_{i j}\left(a_{2}+2 a_{4}\right) M_{W}^{2} R^{2}\right] .
\end{aligned}
$$

Now consider the tauon partial decay width ratio $\Gamma(\tau \rightarrow \mu \bar{\nu} \nu) / \Gamma(\tau \rightarrow e \bar{\nu} \nu)$, using the current experimental limit [19] we find

$$
2 M_{W}^{2} R^{2}\left[a_{1}^{\tau \mu}+a_{3}^{\tau \mu}-a_{1}^{\tau e}-a_{3}^{\tau e}-\frac{m_{\mu}}{m_{\tau}}\left(a_{2}^{\tau \mu}+2 a_{4}^{\tau \mu}\right)\right] \leq .003
$$

which is a constraint on chiral fermion geography.

For a simple illustration, we use the example of a diagonal charged lepton mass matrix as given in [5]. Actually this kind of mass matrix is unnatural in this setup. However it can be achieved by pairing the left-handed and right-handed leptons in the same generation into a cluster which is well separated from the other two generations' clusters. Choosing this setting, the flavor violating gauge coupling are highly suppressed and the Yukawa coupling of KK Higgs is proportional to charged lepton mass, so

$$
a_{2}, a_{3}, a_{4} \sim 0 .
$$

The universality breaking now is solely from the $y$-dependent couplings of KK $W$. Since the Gaussian width $\sigma$ is much smaller than the radius $R$, the exponential suppression factor can be ignore to a good approximation. The series can be summed [20] and keeping the lowest order in $y /(\pi R) \ll 1$ we get

$$
\begin{aligned}
a_{1}^{\tau \mu} \sim 2 \sum_{n=1} \frac{1}{n^{2}} \cos \frac{n y_{\tau}^{L}}{R} \cos \frac{n y_{\mu}^{L}}{R}=\frac{\pi^{2}}{6}\left[2-3 \frac{\left|y_{\tau}^{L}+y_{\mu}^{L}\right|}{R \pi}-3 \frac{\left|y_{\tau}^{L}-y_{\mu}^{L}\right|}{R \pi}\right]+\mathcal{O}\left(\frac{y^{2}}{\pi^{2} R^{2}}\right) \\
a_{1}^{\tau e} \sim 2 \sum_{n=1} \frac{1}{n^{2}} \cos \frac{n y_{\tau}^{L}}{R} \cos \frac{n y_{e}^{L}}{R}=\frac{\pi^{2}}{6}\left[2-3 \frac{\left|y_{\tau}^{L}+y_{e}^{L}\right|}{R \pi}-3 \frac{\left|y_{\tau}^{L}-y_{e}^{L}\right|}{R \pi}\right]+\mathcal{O}\left(\frac{y^{2}}{\pi^{2} R^{2}}\right)
\end{aligned}
$$


We have the limit

$$
M_{W}^{2} R \pi\left(\left|y_{\tau}^{L}+y_{e}^{L}\right|+\left|y_{\tau}^{L}-y_{e}^{L}\right|-\left|y_{\tau}^{L}+y_{\mu}^{L}\right|-\left|y_{\tau}^{L}-y_{\mu}^{L}\right|\right)<0.003 .
$$

If we choose $y_{\tau}^{L}=0$ and $R=300 \mathrm{GeV}^{-1}$ then we have

$$
\frac{\left|y_{e}^{L}\right|-\left|y_{\mu}^{L}\right|}{R}<6.6 \times 10^{-3}\left(\frac{R^{-1}}{300 \mathrm{GeV}}\right)^{2}
$$

which give limits on the separation between different generations.

We emphasize that even for this extreme case the fermion location dependence still breaks the charged lepton universality. It is a generic feature of this model. For more general non-diagonal mass matrices such as that studied in [21] [22] the $\mathrm{KK} \mathrm{Z}$ and $h^{+}$will also contribute to the breaking of universality. We note that the charged lepton matrix of [22] cannot be easily incorporated in the split fermion scenario although it has other success.

We see from the above that flavor violating neutral currents are generic in this scenario. In next section we will discuss the constraint from flavor violating reactions.

\section{2 $\mu$ and $\tau$ to three charged leptons}

Due to the existence of flavor violating interactions in the gauge and the Higgs sectors, the following processes l: $\mu \rightarrow 3 e, \tau \rightarrow 3 e, \tau \rightarrow \mu e e, \tau \rightarrow \mu \mu e$ and $\tau \rightarrow 3 \mu$ will be induced by virtual KK Z, photon, scalar and pseudoscalar boson exchange at tree level as shown in Fig.2. The present upper branch ratio limit for the muon is around $10^{-12}$ and for the $\tau$ is about $10^{-6}$ [19].

The low energy effective Lagrangian for $\mu \rightarrow 3 e$ is easily calculated to be

$$
\sum_{n=1}\left\{\frac{M_{\chi_{n}^{0}}^{2}-M_{h_{n}^{0}}^{2}}{M_{h_{n}^{0}}^{2} M_{\chi_{n}^{0}}^{2}} \lambda_{e e, n}^{L R} \lambda_{e \mu, n}^{L R}\left(\bar{e}_{L} e_{R}\right)\left(\bar{e}_{L} \mu_{R}\right)+\frac{M_{\chi_{n}^{0}}^{2}+M_{h_{n}^{0}}^{2}}{M_{h_{n}^{0}}^{2} M_{\chi_{n}^{0}}^{2}} \lambda_{e e, n}^{L R *} \lambda_{e \mu, n}^{L R}\left(\bar{e}_{R} e_{L}\right)\left(\bar{e}_{L} \mu_{R}\right)\right.
$$



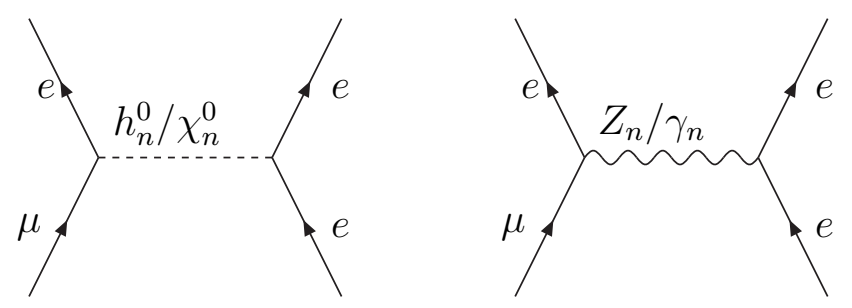

Figure 2: Diagrams for $\mu$ decays into 3 electrons.

$$
\begin{array}{r}
+\frac{M_{\chi_{n}^{0}}^{2}-M_{h_{n}^{0}}^{2}}{M_{h_{n}^{0}}^{2} M_{\chi_{n}^{0}}^{2}} \lambda_{e e, n}^{L R *} \lambda_{\mu e, n}^{L R *}\left(\bar{e}_{R} e_{L}\right)\left(\bar{e}_{R} \mu_{L}\right)+\frac{M_{\chi_{n}^{0}}^{2}+M_{h_{n}^{0}}^{2}}{M_{h_{n}^{0}}^{2} M_{\chi_{n}^{0}}^{2}} \lambda_{e e, n}^{L R} \lambda_{\mu e, n}^{L R *}\left(\bar{e}_{L} e_{R}\right)\left(\bar{e}_{R} \mu_{L}\right) \\
-\frac{g_{2}^{2}}{\cos ^{2} \theta_{W}} \frac{1}{M_{Z, n}^{2}}\left[\bar{e} \gamma^{\mu}\left(g_{L}\left(U_{e e}^{L, n}\right)^{*} \hat{L}+g_{R}\left(U_{e e}^{R, n}\right)^{*} \hat{R}\right) e\right]\left[\bar{e} \gamma_{\mu}\left(g_{L} U_{e \mu}^{L, n} \hat{L}+g_{R} U_{e \mu}^{R, n} \hat{R}\right) \mu\right] \\
\left.-\frac{e^{2}}{M_{\gamma, n}^{2}}\left[\bar{e} \gamma^{\mu}\left(\left(U_{e e}^{L, n}\right)^{*} \hat{L}+\left(U_{e e}^{R, n}\right)^{*} \hat{R}\right) e\right]\left[\bar{e} \gamma_{\mu}\left(U_{e \mu}^{L, n} \hat{L}+U_{e \mu}^{R, n} \hat{R}\right) \mu\right]\right\}+h . c .(20)
\end{array}
$$

the sign difference in front of pseudoscalar term is due to the nature of its imaginary coupling. One can see that the terms $\left(\bar{e}_{L} e_{R}\right)\left(\bar{e}_{L} \mu_{R}\right)$ and $\left(\bar{e}_{R} e_{L}\right)\left(\bar{e}_{R} \mu_{L}\right)$ are almost vanishing because KK scalar and KK pseudoscalar are nearly degenerated if we assume that the SM Higgs mass is not too much heavier than the $W$ boson mass. For the high KK states we can ignore these masses.

To a good approximation we can neglect final state lepton masses and obtain the branching ratio:

$$
B(\mu \rightarrow 3 e)=\frac{\Gamma(\mu \rightarrow 3 e)}{\Gamma(\mu \rightarrow e \bar{\nu} \nu)} \sim 2 M_{W}^{4} R^{4}\left[s_{L L}^{2}+s_{R R}^{2}+2 s_{L R}^{2}+2 s_{R L}^{2}+4 v_{R R}^{2}+4 v_{L L}^{2}\right]
$$

where

$$
\begin{aligned}
& v_{L L}=\left(\sin ^{2} \theta_{W}+\frac{g_{L}^{2}}{\cos ^{2} \theta_{W}}\right) \sum_{n=1} \frac{1}{n^{2}}\left(U_{e e}^{L(n)}\right)^{*} U_{e \mu}^{L(n)}, \\
& v_{R R}=\left(\sin ^{2} \theta_{W}+\frac{g_{R}^{2}}{\cos ^{2} \theta_{W}}\right) \sum_{n=1} \frac{1}{n^{2}}\left(U_{e e}^{R(n)}\right)^{*} U_{e \mu}^{R(n)},
\end{aligned}
$$




$$
\begin{gathered}
s_{L L}=-\sum_{n=1} \frac{\lambda_{\mu e, n}^{L R *} \lambda_{e e, n}^{L R}}{g_{2}^{2} n^{2}}, \quad s_{R R}=-\sum_{n=1} \frac{\lambda_{e \mu, n}^{L R} \lambda_{e e, n}^{L R *}}{g_{2}^{2} n^{2}}, \\
s_{L R}=-\left(\sin ^{2} \theta_{W}+\frac{g_{L} g_{R}}{\cos ^{2} \theta_{W}}\right) \sum_{n=1} \frac{1}{n^{2}}\left(U_{e e}^{L(n)}\right)^{*} U_{e \mu}^{R(n)}, \\
s_{R L}=-\left(\sin ^{2} \theta_{W}+\frac{g_{L} g_{R}}{\cos ^{2} \theta_{W}}\right) \sum_{n=1} \frac{1}{n^{2}}\left(U_{e e}^{R(n)}\right)^{*} U_{e \mu}^{L(n)} .
\end{gathered}
$$

Assuming a universal Yukawa coupling, $f=1$, and again we take the nearly diagonal mass matrix as an example. Then the flavor violating coupling will only appear in Higgs sector. It predicts $v_{L L}, v_{R R}, s_{L R}, s_{R L} \sim 0$ and

$$
\begin{aligned}
\lambda_{e e, n}^{L R} & \sim \frac{g_{2} m_{e}}{\sqrt{2} M_{W}} \cos \frac{n \bar{y}_{e e}^{L R}}{R}, \\
\lambda_{e \mu, n}^{L R} & \sim \exp \left(-\frac{\left(\triangle_{e \mu}^{L R}\right)^{2}}{4 \sigma^{2}}\right) \cos \frac{n \bar{y}_{e \mu}^{L R}}{R} .
\end{aligned}
$$

So in this case,

$$
s_{L L} \sim-\frac{\pi^{2}}{6} \frac{m_{e}}{\sqrt{2} M_{W} g_{2}} \exp \left(-\frac{\left(\triangle_{21}^{L R}\right)^{2}}{4 \sigma^{2}}\right), \quad s_{R R} \sim-\frac{\pi^{2}}{6} \frac{m_{e}}{\sqrt{2} M_{W} g_{2}} \exp \left(-\frac{\left(\triangle_{12}^{L R}\right)^{2}}{4 \sigma^{2}}\right) .
$$

Taking $R^{-1} \sim 300 \mathrm{GeV}$, a value within the bound universal bulk models we obtain

$$
B(\mu \rightarrow 3 e) \sim 2.6 \times 10^{-12}\left[\exp \left(-\frac{\left(\triangle_{12}^{L R}\right)^{2}}{2 \sigma^{2}}\right)+\exp \left(-\frac{\left(\triangle_{12}^{R L}\right)^{2}}{2 \sigma^{2}}\right)\right]\left(\frac{300 \mathrm{GeV}}{R^{-1}}\right)^{4}
$$

which is not far from the experimental limit. The branching is also a very sensitive probe of the compactification radius $R$. Similar expressions can be derived for $\tau$ flavor violating decays which is also much lower than current experimental limits.

For $B\left(\tau^{-} \rightarrow e^{+} e^{-} \mu^{-}\right)$and $B\left(\tau^{-} \rightarrow e^{-} \mu^{-} \mu^{+}\right)$the formula is slightly different since there no identical particles in the final states.

$$
B\left(\tau^{-} \rightarrow e^{+} e^{-} \mu^{-}\right) \sim\left(2 M_{W}^{2} R^{2}\right)^{2}\left[s_{L L}^{2}+s_{R R}^{2}+s_{L R}^{2}+s_{R L}^{2}+v_{R R}^{2}+v_{L L}^{2}\right]
$$


with replaced flavor indices $\mu e \rightarrow \tau e$ and $e e \rightarrow \mu e$ in Eq.(31).

We want to stress again we have used a special diagonal mass matrix to indicate what can be learned. In general, the flavor violating process if observed will set constraint on how far the leptons should be away from each other and Eqs.(30) and (31) should be used. But than numerical method is needed to solve them.

Without a theoretical understanding of the dynamics that determines the locations of the fermions, we do a brute force numerical study. A Monte Carlo program is composed to scan the possible mass matrices which can be accommodated in this model and also satisfy the observed charged lepton masses. Then the rotation matrices $V^{L}$ and $V^{R}$ are calculated for each set of the lepton locations. These in turn are used to calculate all the coupling vertices of KK bosons. Of the half million of mass matrices we scanned many contained off diagonal elements. However, only a small fraction $(<5 \%)$ of these can pass the experimental bound of $B(\mu \rightarrow 3 e)<10^{-12}$. For those satisfying the rare decays limit we use their parameters to calculate the Michel parameter $\eta$. Setting the ratio $(\sigma / R)=1 / 50$, the numerical results give $|\eta| \leq 3 \times 10^{-6}\left(300 \mathrm{GeV} / R^{-1}\right)^{2}$. This will present a formidable experimental challenge. There is a direct correlation between $\eta$ and $\mu \rightarrow 3 e$ since the KK $Z$ and Higgs exchanges that will affect $\eta$ also contribute to the rare decay process. The stringent experimental constrain for the rare decay mode sets the upper limit for $\eta$. We note

in passing that the situation for $\tau$ decays is more hopeful since the constraint from its rare decays are less severe.

\subsection{Muonium-Antimuonium Conversion}

Unlike the rare decays discussed before, here both $(V \pm A)^{2}-$ and $(V \pm A)(V \mp A)-$ type lepton number violating interactions are present at the same time. The effective 


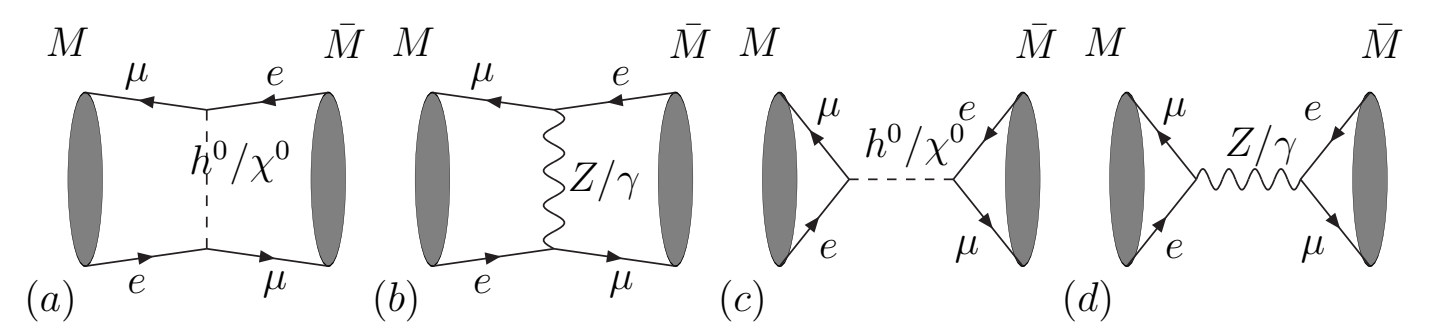

Figure 3: Diagrams of Muonium-Antimuonium Conversion

Hamiltonian for the conversion is given by [23],

$$
\mathcal{H}=\frac{\mathcal{G}}{\sqrt{2}}\left(\bar{\mu} \gamma^{\mu}\left(1 \pm \gamma^{5}\right) e\right)\left(\bar{\mu} \gamma_{\mu}\left(1 \pm \gamma^{5}\right) e\right)+\frac{\mathcal{F}}{\sqrt{2}}\left(\bar{\mu} \gamma^{\mu}\left(1 \pm \gamma^{5}\right) e\right)\left(\bar{\mu} \gamma_{\mu}\left(1 \mp \gamma^{5}\right) e\right)
$$

. The muonium-antimuonium transition matrix elements involve different hyperfine states listed below:

$$
\begin{aligned}
\triangle_{(1, \pm 1)} & =<\bar{M}_{1, \pm 1}|\mathcal{H}| M_{1, \pm 1}>=\frac{16}{\sqrt{2} \pi a_{0}^{3}}\left(\mathcal{G}-\frac{1}{4} \mathcal{F}\right) \\
\triangle_{(1,0)} & =<\bar{M}_{1,0}|\mathcal{H}| M_{1,0}>=\frac{16}{\sqrt{2} \pi a_{0}^{3}}\left(\mathcal{G}-\frac{1}{4} \mathcal{F}\right) \\
\triangle_{(0,0)} & =<\bar{M}_{0,0}|\mathcal{H}| M_{0,0}>=\frac{16}{\sqrt{2} \pi a_{0}^{3}}\left(\mathcal{G}+\frac{3}{4} \mathcal{F}\right)
\end{aligned}
$$

where $a_{0}$ is the Bohr radius of the muonium $\left(m_{r} \alpha\right)^{-1}$ with $m_{r}^{-1}=m_{\mu}^{-1}+m_{e}^{-1}$. Assuming that each state is produced with equal probability initially the integrated probability of a muonium to antimuonium conversion is

$$
\begin{aligned}
P_{M \bar{M}} & =64^{3}\left(\frac{2 \pi^{2} \alpha^{3}}{G_{F} m_{\mu}^{2}}\right)^{2}\left(\frac{m_{e}}{m_{\mu}}\right)^{6}\left(\frac{\mathcal{G}^{2}+\frac{3}{16} \mathcal{F}^{2}}{G_{F}^{2}}\right) \\
& \simeq 2.5 \times 10^{-5}\left(\frac{\mathcal{G}^{2}+\frac{3}{16} \mathcal{F}^{2}}{G_{F}^{2}}\right) .
\end{aligned}
$$


The current experimental limit for this process is $P_{M \bar{M}} \leq 8.3 \times 10^{-11}$ 24 which implies

$$
\sqrt{\mathcal{G}^{2}+\frac{3}{16} \mathcal{F}^{2}}<3.0 \times 10^{-3} G_{F} \quad(90 \% \text { C.L. }) .
$$

Taking diagonal mass matrix as an example again, the flavor violating interaction is mediated only by KK Higgs. Assume that $R \sim 300 \mathrm{GeV}^{-1}$ the above limit will translate into the following bound:

$$
\frac{\left(\triangle_{12}^{L R}\right)^{2}+\left(\triangle_{12}^{R L}\right)^{2}}{\sigma^{2}}>13.8
$$

\section{$4 \quad W$ boson universality in the $\mathbf{S M}$}

As we have seen previously that the LFV mechanism predicts that universality holds in $W$ boson decays but not $\mu$ or $\tau$ decays. Thus, it is important to establish the SM values for these processes. We calculate the $W$ boson branching ratios to 1-loop order in the on-shell scheme and used the unitary gauge which was done in [13]. It is well known the width of $W \rightarrow l \nu$ is infrared finite only after including the radiative mode $W \rightarrow l \nu \gamma$ [25]. After a laborious calculation we find the leptonic decay width including undetected photon is

$$
\begin{aligned}
\frac{\Gamma}{\Gamma^{0}}=\left[1+\frac{\alpha}{2 \pi}\left\{2\left(2+\frac{1+\beta}{1-\beta} \ln \beta\right)\right.\right. & \left(\ln \frac{M_{W}}{2 \triangle E_{l}}+2 \ln (1-\beta)\right) \\
& \left.\left.-\frac{3}{2}(1-\beta) \ln \beta+f_{H} \beta\right\}\right]
\end{aligned}
$$

where $\beta \equiv m_{l}^{2} / M_{W}^{2}$ and $\Gamma^{0}=g_{2}^{2} M_{W}^{2}(2+\beta)(1-\beta) / 6$ is the lowest order width. The quantity $\triangle E_{l}$ is the finite energy resolution of the charged lepton and is determined by a given experiment. $f_{H}$ is a complicate function dependent on the Higgs mass. 
The exact form of $f_{H}$ is not very illuminating and to a good approximation it is

$$
f_{H} \cong 7.72+0.78 \ln \frac{M_{W}^{2}}{M_{H}^{2}} .
$$

Numerically the values of $f_{H}$ are $\{7.36,6.32,5.84,5.34\}$ corresponding to Higgs masses of $M_{H}=\{110,180,250,400\} \mathrm{GeV}$ respectively. Assuming that energy resolutions are the same for all charged leptons the $W \rightarrow \nu e, \nu \mu, \nu \tau$ decay width ratio is $1: 1.067: 1.103$ for $\triangle E=2 \mathrm{GeV}$ and $1: 1.038: 1.057$ for $\triangle E=5 \mathrm{GeV}$. With the expected large number of $W$ bosons to be produced at the LHC[26] we can expect this prediction to be tested in the near future.

\section{Conclusion}

We have shown that in the split fermion scenario with a bulk Higgs boson it is not possible to diagonalize simultaneously the lepton mass matrix and the Yukawa matrix of the KK Higgs modes. This complements the FCNC arising from KK excitations of the $Z$ boson and the photon. This leads to interesting new mechanism for rare $\mu$ and $\tau$ decays at the tree level without affecting lepton universality as probe by $W$ boson decays. On the other hand leptonic universality as probed by $\tau$ lepton decays is altered by the virtual KK gauge boson and KK Higgs exchanges. This gives a upper limit on the separation of different families of leptons. In contrast rare LFV effects if seen are to be understood as measuring the relative distances of a left-handed fermion of one family to the right-handed fermion of a different family in the extra dimension. If no signals are found in the next round of experiments they give a lower bound on the fermion separations. Similarly the fermion masses sets the relative distances between fermions of opposite chiralities in the same family. 
Another interesting difference the split fermion scenario as compare to usual extra dimension models is the non-universality of the KK gauge bosons coupling the electron, muon and $\tau$. In other words when a KK gauge boson, for instance the $n=1 \mathrm{KK}$ photon, is produced at high energies we expect its decay widths into electrons, muons and taus to be different since the $U$ matrices of Eq.(19) are sensitive to the family indices. This will lead to an apparent charge violation of electric charge universality.

The above considerations can easily be extended to the quark sector. The universality test of pion leptonic decay will set a limit of ( see Eq.(24) )

$$
M_{W}^{2} R^{2} \sum_{n=1} \frac{1}{n^{2}} \operatorname{Re}\left[U_{u d}^{L(n)}\left(U_{11}^{L(n) *}-U_{22}^{L(n) *}\right)\right] \lesssim 10^{-7}
$$

assuming that it is dominated by flavor conserving KK $W$ exchanging and $U_{u d}^{L(n)}$ is an obvious generalization to the quark sector. In general we do not expect universality to hold when comparing effective charged current strengths as measured in semileptonic versus leptonic experiments. This opens up a new way of looking at the Cabibbo universality which we leave for future considerations.

The split fermion scenario suffers from a number of drawbacks. On the theoretical side there is a lack of understanding of the dynamics that determines the different chiral fermion locations and the form of the wavefunctions. Phenomenologically there are many parameters all of which has to be fixed by experiments. Despite all of these flaws some general distinguishing features emerged. Among them is non-universality in both charged and neutral currents sectors as probed by experiments done on the brane. On the other hand, the level at which these effects are expected depends on the details of the model.

Our study has shown the importance of low energy precision tests in covering 
the parameter space for these models. While it is too early to do complete phenomenological analysis of even the minimal model due to the scarcity of data at the same time we feel that more studies involving similar rare processes are crucial. They are complementary to direct collider searches for the KK excitations of the SM particles.

This work is supported in part by the Natural Science and Engineering Council of Canada. We wish to thank Professor D. Chang for the kind hospitality at the National Center of Theoretical Science where this work was completed. 


\section{A Feynman Rules}

Combining all pieces discussed in Sec 2 together, the relevant terms of the 5D SM are:

$$
\begin{aligned}
\mathcal{L}_{5}+\mathcal{L}_{G F}= & -\frac{1}{4}\left(\partial_{M} P_{N}-\partial_{N} P_{M}\right)^{2}-\frac{1}{4}\left(\partial_{M} Z_{N}-\partial_{N} Z_{M}\right)^{2} \\
& -\frac{1}{2}\left(\partial_{M} W_{N}^{+}-\partial_{N} W_{M}^{+}\right)\left(\partial^{M} W^{-, N}-\partial^{N} W^{-, M}\right) \\
& +\frac{1}{2}\left(\partial^{M} \phi^{0}\right)\left(\partial_{M} \phi^{0 *}\right)+\left(\partial^{M} h^{+}\right)\left(\partial_{M} h^{-}\right) \\
& +\frac{1}{4}\left(g v_{0} c\right)^{2} W_{M}^{+} W^{-, M}+\frac{1}{8}\left(g v_{0}\right)^{2} Z_{M} Z^{M} \\
& -\frac{1}{\xi}\left(\partial^{M} W_{M}^{+}\right)\left(\partial^{N} W_{N}^{-}\right)-\xi\left(\frac{g v_{0} c}{2}\right)^{2} h^{+} h^{-} \\
& -\frac{1}{2 \eta}\left(\partial^{M} Z_{M}\right)^{2}-\frac{\eta}{2}\left(\frac{g v_{0}}{2}\right)^{2}\left(\chi^{0}\right)^{2}-\frac{1}{2 \alpha}\left(\partial^{M} P_{M}\right)^{2}+\cdots
\end{aligned}
$$

where $P$ represents photon, $\phi^{0}=h^{0}+i \chi^{0}$ and $v_{0}=\sqrt{2 \pi R} v_{b}^{\frac{3}{2}}$.

Employing the KK decomposition, imposing the appropriate boundary conditions and integrating over $y$, we get the $4 \mathrm{D}$ effective Lagrangian

$$
\begin{aligned}
& \mathcal{L}_{4}=-\frac{1}{4} \sum_{n=0} P_{n}^{\mu \nu} P_{n, \mu \nu}+\frac{1}{2} \sum_{n=1}\left[\left(\partial^{\mu} P_{n}^{4}\right)^{2}+\frac{n^{2}}{R^{2}}\left(P_{n}\right)^{2}-2 \frac{n}{R}\left(\partial^{\mu} P_{n}^{4}\right) P_{n, \mu}\right] \\
& -\frac{1}{2 \alpha} \sum_{n=0}\left[\left(\partial_{\mu} P_{n}^{\mu}\right)^{2}+2 \frac{n}{R} \partial_{\mu} P_{n}^{\mu} P_{n}^{4}+\frac{n^{2}}{R^{2}}\left(P_{n}^{4}\right)^{2}\right] \\
& -\frac{1}{4} \sum_{n=0} Z_{n}^{\mu \nu} Z_{n, \mu \nu}+\frac{1}{2} \sum_{n=1}\left[\left(\partial^{\mu} Z_{n}^{4}\right)^{2}+\frac{n^{2}}{R^{2}}\left(Z_{n}\right)^{2}-2 \frac{n}{R}\left(\partial^{\mu} Z_{n}^{4}\right) Z_{n, \mu}\right] \\
& -\frac{1}{2 \eta} \sum_{n=0}\left[\left(\partial_{\mu} Z_{n}^{\mu}\right)^{2}+2 \frac{n}{R} \partial_{\mu} Z_{n}^{\mu} Z_{n}^{4}+\frac{n^{2}}{R^{2}}\left(Z_{n}^{4}\right)^{2}\right] \\
& +\frac{\left(g v_{0}\right)^{2}}{8}\left[Z_{0, \mu} Z_{0}^{\mu}+\sum_{n=1}\left(Z_{n, \mu} Z_{n}^{\mu}-Z_{n}^{4} Z_{n}^{4}\right)\right]-\frac{1}{2} \sum_{n=0} W_{n}^{\mu \nu+} W_{n, \mu \nu}^{-} \\
& +\sum_{n=1}\left[\left(\partial^{\mu} W_{n}^{4+} \partial_{\mu} W_{n}^{4-}\right)+\frac{n^{2}}{R^{2}}\left(W_{n \mu}^{+} W_{n}^{\mu-}\right)-\frac{n}{R}\left(\partial^{\mu} W_{n}^{4-} W_{n \mu}^{+}+\partial^{\mu} W_{n}^{4+} W_{n \mu}^{-}\right)\right]
\end{aligned}
$$




$$
\begin{aligned}
& -\frac{1}{\xi} \sum_{n=0}\left[\left(\partial_{\mu} W_{n}^{\mu+}\right)\left(\partial_{\mu} W_{n}^{\mu-}\right)+\frac{n}{R}\left(\partial_{\mu} W_{n}^{\mu+} W_{n}^{4-}+\partial_{\mu} W_{n}^{\mu-} W_{n}^{4+}\right)+\frac{n^{2}}{R^{2}}\left(W_{n}^{4+} W_{n}^{4-}\right)\right] \\
& +\frac{\left(g v_{0} c\right)^{2}}{4}\left[W_{0, \mu}^{+} W_{0}^{-, \mu}+\sum_{n=1}\left(W_{n, \mu}^{+} W_{n}^{-, \mu}-W_{n}^{4+} W_{n}^{4-}\right)\right] \\
& +\frac{1}{2} \sum_{n=0}\left[\left(\partial^{\mu} h_{n}^{0}\right)^{2}-\frac{n^{2}}{R^{2}}\left(h_{n}^{0}\right)^{2}\right]+\frac{1}{2} \sum_{n=0}\left[\left(\partial^{\mu} \chi_{n}^{0}\right)^{2}-\left(\eta\left(\frac{g v_{0}}{2}\right)^{2}+\frac{n^{2}}{R^{2}}\right)\left(\chi_{n}^{0}\right)^{2}\right] \\
& +\sum_{n=0}\left[\partial^{\mu} h_{n}^{+} \partial_{\mu} h_{n}^{-}-\left(\xi\left(\frac{g v_{0} c}{2}\right)^{2}+\frac{n^{2}}{R^{2}}\right) h_{n}^{+} h_{n}^{-}\right]+\cdots
\end{aligned}
$$

From the above expansion, the propagators can be read:

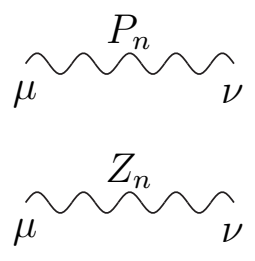

$$
\begin{aligned}
& \frac{-i}{p^{2}-\frac{n^{2}}{R^{2}}}\left\{g^{\mu \nu}+\frac{(\alpha-1) p^{\mu} p^{\nu}}{p^{2}-\alpha \frac{n^{2}}{R^{2}}}\right\} \\
& \frac{-i}{p^{2}-\left(M_{Z}^{2}+\frac{n^{2}}{R^{2}}\right)}\left\{g^{\mu \nu}+\frac{(\eta-1) p^{\mu} p^{\nu}}{p^{2}-\eta\left(M_{Z}^{2}+\frac{n^{2}}{R^{2}}\right)}\right\}
\end{aligned}
$$

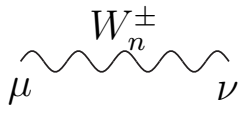

$$
\frac{-i}{p^{2}-\left(M_{W}^{2}+\frac{n^{2}}{R^{2}}\right)}\left\{g^{\mu \nu}+\frac{(\xi-1) p^{\mu} p^{\nu}}{p^{2}-\xi\left(M_{W}^{2}+\frac{n^{2}}{R^{2}}\right)}\right\}
$$

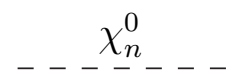

$$
\begin{array}{cc}
\frac{i}{p^{2}-\left(\eta M_{Z}^{2}+\frac{n^{2}}{R^{2}}\right)} & ---\frac{W_{n}^{ \pm 4}}{-}-- \\
\frac{i}{p^{2}-\left(\xi M_{W}^{2}+\frac{n^{2}}{R^{2}}\right)} & ---Z_{n}^{4}--
\end{array}
$$$$
\frac{i}{p^{2}-\left(M_{W}^{2}+\frac{n^{2}}{\xi R^{2}}\right)}
$$

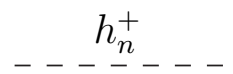

$$
\frac{i}{p^{2}-\left(\xi M_{W}^{2}+\frac{n^{2}}{R^{2}}\right)}
$$$$
\frac{i}{p^{2}-\left(M_{Z}^{2}+\frac{n^{2}}{\eta R^{2}}\right)}
$$

$h_{n}^{0}$

$$
\frac{i}{p^{2}-\left(m_{H}^{2}+\frac{n^{2}}{R^{2}}\right)}
$$

$P_{n}^{4}$

$$
\frac{i}{p^{2}-\frac{n^{2}}{\alpha R^{2}}}
$$

For the Feynman gauge used in this calculation we set $\alpha=\eta=\xi=1$ otherwise there appear the following mixing vertex

$$
\begin{aligned}
& P_{n}^{\mu} \sim \boldsymbol{p}_{p}^{--} P_{n}^{4} \quad-\frac{n}{R} \frac{1-\alpha}{\alpha} p^{\mu} \\
& Z_{n}^{\mu} \sim \boldsymbol{x}_{p}^{--} Z_{n}^{4} \quad-\frac{n}{R} \frac{1-\eta}{\eta} p^{\mu} \\
& W_{n}^{ \pm \mu} \leadsto \times-{ }_{p}-W_{n}^{ \pm 4} \quad-\frac{n}{R} \frac{1-\xi}{\xi} p^{\mu}
\end{aligned}
$$


The brane or zero mode fermion couplings in terms of mass eigenstates are summarized in the following figure, where $\hat{R} / \hat{L}=\frac{1}{2}\left(1 \pm \gamma^{5}\right)$ and summation is understood for the repeating indices.

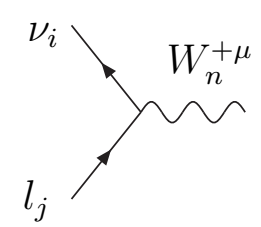

$$
\frac{i g_{2}}{\sqrt{2}} K_{n} \exp \left[-\frac{n^{2} \sigma^{2}}{4 R^{2}}\right] \gamma^{\mu}\left[\left(V^{L}\right)_{i k}^{\dagger} \cos \frac{n y_{k}^{L}}{R} V_{k j}^{L} \hat{L}\right]
$$

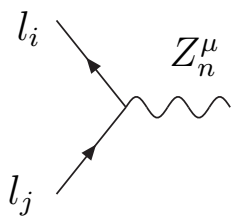

$$
\frac{i g_{2}}{\cos \theta_{W}} K_{n} \exp \left[-\frac{n^{2} \sigma^{2}}{4 R^{2}}\right] \gamma^{\mu}\left[g_{L}\left(V^{L}\right)_{i k}^{\dagger} \cos \frac{n y_{k}^{L}}{R} V_{k j}^{L} \hat{L}+g_{R}\left(V^{R}\right)_{i k}^{\dagger} \cos \frac{n y_{k}^{R}}{R} V_{k j}^{R} \hat{R}\right]
$$

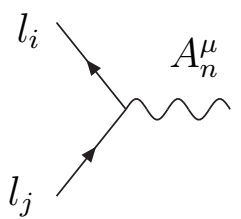

$$
-i e K_{n} \exp \left[-\frac{n^{2} \sigma^{2}}{4 R^{2}}\right] \gamma^{\mu}\left[\left(V^{L}\right)_{i k}^{\dagger} \cos \frac{n y_{k}^{L}}{R} V_{k j}^{L} \hat{L}+\left(V^{R}\right)_{i k}^{\dagger} \cos \frac{n y_{k}^{R}}{R} V_{k j}^{R} \hat{R}\right]
$$

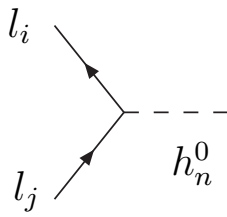

$$
-i \frac{K_{n}}{\sqrt{2}}\left[\frac{1}{2}\left(\lambda_{i j, n}^{L R}+\lambda_{j i, n}^{L R *}\right)+\frac{1}{2}\left(\lambda_{i j, n}^{L R}-\lambda_{j i, n}^{L R *}\right) \gamma^{5}\right]
$$

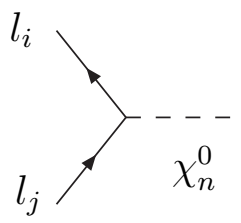

$$
\frac{K_{n}}{\sqrt{2}}\left[\frac{1}{2}\left(\lambda_{i j, n}^{L R}-\lambda_{j i, n}^{L R *}\right)+\frac{1}{2}\left(\lambda_{i j, n}^{L R}+\lambda_{j i, n}^{L R *}\right) \gamma^{5}\right]
$$

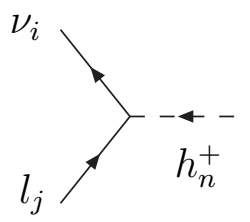

$$
-i \sqrt{2} K_{n} \lambda_{i j, n}^{L R} \hat{R}
$$

where $K_{n}=\delta_{n, 0}+\sqrt{2}\left(1-\delta_{n, 0}\right)$ and $\lambda_{i j, n}^{L R}=\exp \left[-\frac{n^{2} \sigma^{2}}{4 R^{2}}\right]\left[\left(V^{L}\right)_{i k}^{\dagger} f_{k l} \exp \left[-\frac{\left(\triangle_{k l}^{L R}\right)^{2}}{4 \sigma^{2}}\right] \cos \frac{n \bar{y}_{k l}^{L R}}{R} V_{l j}^{R}\right]$. One can check that when $n=0$ they reduce to the usual flavor diagonal SM couplings. Note that in general for $n \geq 1, \lambda_{i j, n}^{L R} \neq \lambda_{j i, n}^{L R *}$. 


\section{References}

[1] N. Arkani-Hamed, S. Dimopoulos and G. Dvali, Phys. Lett. B429, 263 (1998) hep-ph/9803315

[2] I. Antoniadis, Phys. Lett. B246, 377 (1990).

[3] J. Lykken, Phys. Rev. D54, 3693 (1996).

[4] N. Arkani-Hamed and M. Schmaltz, Phys. Rev. D61, 033005 (2000) [hep$\mathrm{ph} / 9903417$

N. Arkani-Hamed, Y. Grossman and M. Schmaltz, Phys. Rev. D61, 115004 (2000) hep-ph/9909411.

[5] E.A. Mirabelli and M. Schmaltz, Phys. Rev. D61, 113011 (2000)

[6] A. Delgado, A. Pomarol and M. Quiros, J. High Energy Phys. 0001,030(2000)

[7] G.C. Branco, A. de Gouvea and M.N. Rebelo, Phys. Lett. B506, 115 (2000)

[8] N. Akani-Hamed, S. Dimopoulos, G. Dvali, and J. March-Russell, hepph $9811448(1998)$

K.R. Dienes,E. Dudas, and T. Gherghetta, Nucl. Phys. B577, 25 (1999)

[9] J.-M. Frère, M.V. Libanov, and S.V. Troitsky, JHEP 0111, 025 (2001)

C.S. Lam, and J.N. Ng, Phys. Rev 64D, 113006 (2001)

C.S. Lam ,ibid, 65D, 053009 (2002)

K.R. Dienes and I. Sarcevic, Phys. Lett. B500, 133 (2001) 
[10] A.E. Faraggi and M.E. Pospelov, Phys. Lett. B458 237 (1999) G.C. McLaughlin and J.N. Ng, ibid B470, 157 (1999); ibid B493, 88 (2000) G.C. McLaughlin and J.N. Ng, Phys. Rev. D63 053002 (2001)

[11] D.E. Kaplan and T.M.P. Tait, JHEP 0111, 051 (2001)

[12] F. del Aguila and J. Santiago, JHEP 0203, 010 (2002)

[13] T. W. Appelquist, J. R. Primack and H. R. Quinn, Phys. Rev. D7, 2998(1973); W. J. Marciano and A. Sirlin, Phys. Rev. D8, 3612(1973).

[14] G. Barenboim, G.C. Branco, A. de Gouvea and M.N. Rebelo, Phys. Rev D64 $073005(2001)$

[15] V.A. Rubakov and M.E Shaposhnikov, Phys. Lett. B125, 136 (1983)

R. Jackiw and C. Rebbi, Phys. Rev. D13, 3398 (1976)

[16] G. Dvali and M. Shifman, Phys. Lett. B475, 295 (2000)

[17] C.D. Carone, Phys. Rev D61, 015008 (2000) T. Appelquist,H.C. Cheng, and B. Dobrescu, ibid D64 035002 (2001)

[18] W. J. Marciano, Phys. Rev. D60, 093006 (1999)

M. Masip and A. Pomarol, Phys. Rev D60 096005 (1999)

[19] D.E. Groom et al, The Euro. Phys. J.C15, 1(2000)

[20] We use the formular $\sum_{n=1}^{\infty} \frac{\cos (n \pi \triangle)}{n^{2}}=\frac{\pi^{2}}{6}\left[1-3|\triangle|+\frac{3}{2} \triangle^{2}\right]$ and has a periodicity of $\triangle=\bmod 2$.

[21] L. Ibáñez and G.G. Ross, Phys. Lett. B332, 100(1994). 
[22] H. Nishiura, K. Matsuda, T. Kikuchi and T. Fukuyama, hep-ph/0202189].

[23] F. Feinberg and S. Weinberg, Phys. Rev. Lett. 6, 381(1961);

K. Horikawa and K. Sasaki, Phys. Rev. D 53, 560(1996)

[24] L. Willmann et al, Phys. Rev. Lett. 82, 49(1999).

[25] T. Kinoshita, J. Math. Phys. 3, 650(1962)

T. D. Lee and M. Nauenberg, Phys. Rev. 133, B1549(1964).

[26] F. Gianotti and M. P. Altarelli, Nucl. Phys. B(Proc. Suppl.)89,177(2000). 